\title{
Botulinum toxin
}

\author{
Useful in adult onset focal dystonias
}

Human botulism, due to the ingestion of toxin produced by Clostridium botulinum, is rare but potentially fatal and causes an acute symmetric descending paralytic illness particularly affecting eye and bulbar muscles. ${ }^{1}$ The toxin paralyses by preventing the release of acetylcholine from nerve terminals - thereby leading to irreversible neuromuscular blockade. Recovery occurs over about three months as axons resprout and new extrajunctional acetylcholine receptors form. Although antitoxins and antibiotics are recommended, there is no known cure.

Botulinum toxin was first used therapeutically to correct squints by altering the balance between muscles responsible for the abnormal position of the eye in the orbit. ${ }^{2}$ Since then it has become the preferred treatment for several adult onset focal dystonias and hemifacial spasm. Indications for its use increase rapidly, and its role is currently being explored in various medical and surgical specialties. The specific local effects of injections with botulinum toxin in inducing temporary muscle weakness have already helped thousands of patients with previously untreatable, often painful, and functionally devastating muscle spasms. Not surprisingly, introduction of this treatment into clinical practice has also stimulated a flurry of sensational media publicity, with headlines ranging from "a little bit of botulinum can do you good" to "lethal nerve poison miraculously makes the blind see and the mute speak."

In the focal dystonias, a poorly understood group of conditions believed to be due to neurochemical abnormalities in the basal ganglia, treatment has been based on the injection of small quantities of botulinum toxin into the locally affected muscles to produce weakness sufficient to eliminate excessive contraction at the same time as preserving function. The clinical effect appears between one and 15 days after injection and in most cases wears off completely between four and 16 weeks. Essential blepharospasm, which usually begins after the fourth decade of life and is characterised by involuntary repetitive closure of the eyelids, leading to impaired visual function, responds extremely well. Botulinum toxin is injected into three or four superficial sites in each orbicularis oculi muscle, and worthwhile temporary improvement occurs in four out of five patients. ${ }^{3}$ Pretarsal forms of blepharospasm and apraxia of eyelid opening do not respond well. Focal bruising and transient partial ptosis are relatively common whereas transient diplopia and mild facial weakness are rare adverse reactions.

Patients with blepharospasm continue to be misdiagnosed as having a primary psychiatric illness, which leads to inappropriate, distressing referral, and some may have developed their dystonia from inappropriate long term administration of neuroleptic drugs (such as antipsychotics, metoclopramide, and prochlorperazine). Some patients have additional lower facial and jaw opening and closing spasms, which may also respond to botulinum toxin, although the results are less good.

Hemifacial spasm due to intermittent synchronous contraction of the ipsilateral facial nerve is another established indication for botulinum toxin. Other drug treatment is ineffective, and the morbidity and unpredictable results of the current recommended neurosurgical approaches are a major drawback. In hemifacial spasm the muscle contractions usually begin around one orbicularis oculi muscle and then spread to affect the brow, lower face, and platysma. Unlike blepharospasm, the movements are invariably unilateral and may continue in sleep. Chewing and talking characteristically aggravate hemifacial spasm; in contrast, blepharospasm is exacerbated by bright light, reading, or watching television. Injection into the affected orbicularis oculi muscle removes the eye spasm in over $90 \%$ of cases, and a further single small injection into the corrugator muscle may also help mid-face spasm. ${ }^{+}$Together with congenital and acquired squints (particularly those that in turn) blepharospasm and hemifacial spasm constitute the only licensed indications for botulinum type A toxin in the United Kingdom.

Spasmodic torticollis - like blepharospasm, another adult onset focal dystonia-also responds well to botulinum toxin, and many neurologists now regard this as the preferred treatment. The condition usually presents in middle age with involuntary torsion or jerky movements of the neck, frequently accompanied by pain and crippling depression. Because of the complexity of the neck musculature the overall results are less good than those after injection into the orbicularis oculi muscle, but neck posture improves by at least $70 \%$ with an even greater improvement in pain relief..$^{5-7}$ The simple rotatory forms involving mainly the sternomastoid on one side and the contralateral posterior cervical muscles do best. Mild neck weakness and transient dysphagia are the commonest side effects. A few patients go into spontaneous remission during long term treatment whereas about $5 \%$ become refractory to serial injections after two or three years.

Other highly promising uses for botulinum toxin are in the treatment of laryngeal dystonia ${ }^{8}$ and hand cramps, including 
writers' and musicians' dystonias, golfers' "yips," and dart players' cramp..$^{910}$ The toxin may also have a role in treating severe spasticity, facilitating physiotherapy by temporarily relieving pain and increased muscle tone, and in dystonic foot cramps. More controversial uses include pain relief in sports injuries and chronic tension headaches, alleviating eye wrinkles in cosmetic surgery, and injecting the puborectalis muscle to treat a special form of constipation anismus. The toxin has also been used to treat palatal myoclonus, stridor, urinary symptoms, and dysthyroid eye disease.

Treatment with botulinum type A toxin has provided welcome relief and hope for patients with adult onset focal dystonias, who may number 30000 in the United Kingdom. Despite its drawbacks, which include the need to repeat the injection three or four times a year and its expense (about $£ 100$ per injection for blepharospasm and $£ 300$ for torticollis), it has become an established treatment in ophthalmological and neurological practice. ${ }^{11}$ Patients have continued to respond with benefit for more than five years, although antibodies to the toxin may develop in the peripheral blood, leading to initial unresponsiveness or late resistance. ${ }^{12}$ Long term effects on muscle function both locally and distant from the site of injection have yet to be determined. Trials of other types of botulinum toxin are under way, and more effective toxins capable of producing longer durations of benefit without inevitably increasing unwanted effects may be developed in the near future.

A J LEES

Consultant Neurologist,

National Hospital for Neurology and Neurosurgery,

Queen Square,

London WCIN 3BG

1 Hutchinson DN. Foodborne botulism. BMf 1992;305:262-5.

2 Scott AB. Botulinum toxin injections into extraocular muscles as an atternative to strabismus surgery. Ophthalmology 1980;87:1044-9.

3 Elston JS, Russell RWR. Effect of treatment with botulinum toxin on neurogenic blepharospasm. $B M F$ 1985;290:1857-9.

4 Elston JS. Botulinum toxin treatment of hemifacial spasm. $f$ Neurol Neurosurg Psychiatry 1986;49:827-9.

5 Tsui JKC, Eisen A, Stoessi AJ, Calne S, Calne DB. Double-blind study of botulinum toxin in spasmodic torticollis. Lancet 1986;ii:245-6.

6 Stell R, Thompson PD, Marsden CD. Botulinum toxin in spasmodic torticollis. $\mathcal{f}$ Neurol Neurosurg Psychiatry 1988;51:920-3.

7 Blackie JD, Lees AJ, Botulinum toxin in the treatment of spasmodic torticollis. 7 Neurol Neurosurg Psychiatry 1990;53:640-3.

8 Brin MF, Blitzer A, Fahn S, Gould W, Lovelace RE. Adductor laryngeal dystonia (spastic dysphonia): treatment with local injections of botulinum toxin. Mov Disord 1989;4:287-96.

9 Cohen LG, Hallett M, Geller BD, Hochberg F. Treatment of focal dystonias of the hand with botulinum toxin injections. I Neurol Neurosurg Psychiatry 1989;52:355-63.

10 Rivest J, Lees AJ, Marsden CD. Writers' cramp: treatment with botulinum toxin injections. Mro Disord 1991;6:55-9.

11 American Academy of Neurology. Assessment: the clinical usefulness of botulinum toxin-A in treating neurologic disorders. Neurology 1990;40:1332-6.

12 Hambleton P, Cohen HE, Palmer BJ, Melling J. Antitoxins and botulinum toxin treatment. BMJ 1992;304:950-60.

\title{
Cardiovascular disease in developing countries
}

\author{
An epidemic that can be prevented
}

Cardiovascular diseases account for about one quarter of all deaths worldwide. In developed countries about half of all deaths are due to cardiovascular disease (principally coronary heart disease and stroke), and the epidemic is being at least partially prevented in some of these. In developing countries the proportion of deaths due to cardiovascular disease is estimated to be about $15 \%$, but with wide variations. The total number of deaths due to cardiovascular disease, however, is thought to be at least as great as in developed countries as about $80 \%$ of all deaths occur in developing countries. ${ }^{1}$

The epidemic of cardiovascular disease is becoming more apparent in developing countries with increases in both the absolute and relative number of deaths. Different countries have different patterns: stroke is a leading cause of death in China, cardiomyopathies and hypertensive heart disease are important in Africa, and in many countries rheumatic heart disease remains common. The upsurge of deaths from cardiovascular disease is specially evident in those parts of Asia and Latin America where progress against the communicable diseases of childhood has been greatest. ${ }^{1}$ In Singapore, for example, death rates from coronary heart disease doubled in the two decades from $1959 .^{2}$

In Latin America over the next 25 years, deaths from cardiovascular disease are expected to number almost five times the projected total of those from infectious and parasitic diseases, compared with roughly equal numbers in the two groups in 1985. These projections emphasise the need for strategic planning to control the emerging non-communicable diseases even though the communicable diseases are still incompletely controlled.

As deaths from cardiovascular disease rise in developing countries age specific death rates are apparently declining, at least in some countries for which reliable information is available. ${ }^{34}$ The reasons for these favourable trends are unclear, and - unfortunately - the mortality data are usually incomplete and rarely validated. ${ }^{5}$ Nevertheless, the emerging epidemic seems to be real and not a result of changes in diagnostic or certification procedures.

The progressive aging of populations has probably been the most important factor, together with the decline in communicable diseases. ${ }^{4}$ The increasing prevalence in developing countries of the major risk factors for heart diseases and stroke, especially among men, will presumably be important in the future. ${ }^{6-8}$ Therapeutic advances have probably had even less impact on cardiovascular diseases in developing countries than in developed countries. Epidemiological studies are urgently required in developing countries, both to document the epidemic and to clarify the contribution of various risk factors as standards of living improve.

The social and economic causes of cardiovascular disease seem to be the same in developing as in developed countries, with urbanisation having an adverse effect. ${ }^{9}$ The underlying and essential cause of cardiovascular disease is an atherogenic and thrombogenic diet, ${ }^{10}$ leading to high rates of hypercholesterolaemia, obesity, hypertension, and diabetes. The high prevalence in many developing countries of smoking, specially in men, and the high relative risks associated with it make smoking the most readily preventable cause of cardiovascular disease. Hypertension is also already common and increasing in urban areas. ${ }^{6}$

Two strategies are available for preventing cardiovascular disease - the population strategy and the high risk strategy. ${ }^{11}$ The population strategy, which aims to reduce the risks for the whole population, is the most likely to be successful in dealing with the underlying social and economic causes. It needs to use a range of health policy instruments, and its preventive interventions must be safe and cost effective. Preventive strategies such as smoking cessation programmes 